\title{
A Proposed Method for Tumour Segmentation in Brain MRI
}

\author{
Harman Kataria \\ Student, Department of CSE, \\ PEC University of Technology, \\ Chandigarh, 160012
}

\author{
Alka Jindal \\ Assistant professor, department of I.T, \\ PEC university of Technology, \\ Chandigarh, 160012
}

\begin{abstract}
This paper deals with efficient medical image segmentation. The proposed method uses more than one scaling parameters such as sigma and epsilon. The proposed method reduces the number of iteration and tales less computational time than the original method. Comparison between original and proposed method is illustrated with the help of graph. It is possible to apply the proposed method to $2 \mathrm{~d}$ as well as $3 \mathrm{~d}$ images.
\end{abstract}

\section{Keywords}

Active Contour, Segmentation, Intensity Inhomogenity, Magnetic Resonance Image (MRI)

\section{INTRODUCTION}

In the current clinical inflation, medical imaging is becoming a very important aspect for numerous applications ranging from diagnosis to treatment. Today, the diagnosis of a patient is rarely done without the use of imaging technology. After the introduction of digital imaging devices in medicine, computerized tissue recognition and classification have become important in research and clinical applications [1]. Segmentation is a challenging task owing to their complexity. Medical Image Segmentation is the task of segmenting image component into the relevant anatomical components. Magnetic Resonance imaging (MRI) is most commonly imaging technique that is used to produce the detailed image of brain and brain stem. Active Contour method is mainly used for image segmentation. It can be categorized in two classes: edge-based models [2], [3] and region based models [4], [5]. The author Jia Di et al [6], proposed a C-V model that makes segmentation better than the other model. Active contour model is used to evolve the curve on the given image in order to detect objects in the image. C-V model divides the entire region into two parts one is inside part and the other is outside part and we can get the edges from the end of curves iteration. Local Region Based Active Contour is a region based method that is insensitive to noise and it is robust against the initial curve placement. It segments nonhomogenous objects. It uses smaller local regions to segment the object rather than the global region [7].

This paper presents the region-based active contour model in the variational level set formulation. This method applies on both homogenous and non homogenous images. Intensity homogeneity occurs due to technical limitation or an artefact introduced by the object and it is mainly present in real images.

After implementation and testing of the following algorithms: Improved level set, Localised Region Based Active contour and Variational Level set formulation [8]. The performance of variational level set was found out to be best amongst all and the comparison metric of these algorithm is referred from Brain MRI: region based segmentation techniques In this paper a model is proposed, which is a variation of variational set level model, peculiarly. We can extract the tumour itself without the background of the image. This can be done with inclusion of properties like solidity and eccentricity. The model takes into account in reduction of iterations and hence increases in the speed.

The paper is organized as follows. In section 2, we first review the existing region-based active contour in variational level set formulation model and their limitation. Section 3 introduced the proposed method. Section 4 shows the experimental results and discussion of proposed method. The paper is summarized in section 5 .

\section{REGION BASED VARIATIONAL}

\section{LEVEL SET FORMULATION METHOD}

Chunming Li et.al [9] proposed a Variational level set formulation method that is a region based method. This algorithm segments the whole image. It overcomes the problem that occurred due to intensity inhomogeneity. In this method we first define Region Scalable fitting (RSF) energy function and localization is performed by two fitting function f1, f2 and $K_{\sigma}$. This algorithm is able to segment inhomogeneous objects. The standard gradient descent method is use to minimize the energy functional. The minimization equation is.

$$
\begin{aligned}
& \varepsilon_{\epsilon}\left(\phi, f_{1}, f_{2}\right)= \\
& \sum_{i=1}^{2} \lambda_{i} \int\left(\int K(x-y)\left|I(y)-f_{i}(x)\right|^{2} M_{i}^{\epsilon}(\phi(y)) d y\right) d x+ \\
& 2 \nabla H \epsilon(\phi x) d x \quad \text { (1) }
\end{aligned}
$$

Where $\mathrm{I}(\mathrm{x})$ is the image intensity at pixel $\mathrm{x}, \mathrm{H}$ is a Heaviside function, $K_{\sigma}$ is a Gaussian kernel defined as:

$K_{\sigma}(u)=\frac{1}{(2 \Pi)^{n / 2} \sigma^{n}} e^{-\|u\|^{2} / 2 \sigma^{2}}$

With a scale parameter $\sigma>0$, f1 and $\mathrm{f} 2$ are two functions centred at pixel as

$$
\begin{aligned}
& f_{1}(x)=\frac{K_{\sigma} *(H \phi(x) I(x)}{K_{\sigma} * H(\phi(x)} \\
& f_{2}(x)=\frac{K_{\sigma^{*}}((1-H(\phi(x))))}{K_{\sigma} *(1-H(\phi(x))}
\end{aligned}
$$


$\lambda_{1}$ and $\lambda_{2}$ are two constants weights. The evolution equation is defined as below.

$\frac{\delta \phi}{\delta t}=\delta\left(\phi(x) \lambda_{1}\left(\int_{\Omega}^{\cdot} K_{\sigma}(x-y)\left|I(y)-f_{1}(x)\right|^{2} d y\right.\right.$

$\left.+\lambda_{2} \int_{\Omega} K_{\sigma}(x-y)\left|I(y)-f_{1}(x)\right|^{2} d y\right)+v \delta(\phi(x) k+$

$\mu\left(\nabla^{2} \phi(x-k)\right)$

$\phi$ is a signed distance function.

The first two terms is a data fitting term [10], these terms are used for driving active contour toward object boundaries. The third and fourth terms are used for smoothening and maintain the regularity of the contour.

\subsection{Shortcomings}

The algorithm we discussed earlier was applied on both homogenous and non homogenous images but there were some limitations that cannot be ignored. This algorithm uses one scale parameter in the RSF energy to corresponding energy. This algorithm segments the whole brain MRI, but it doesn't distinguish the tumour separately. But for the estimation of volume the tumour has to be diagnosed separately. The future scope of the algorithm as mentioned by the author is to use more than one scale parameters. In this method only one scale parameter $\sigma$ is used. To improve the accuracy and robustness we can use more than one scale parameters. It also increases the computational efficiency of the above mentioned algorithm.

\section{PROPOSED MODEL}

Due to the limitation of the algorithm that was discussed previously, there is a need of improved algorithm that overcomes these limitations. The proposed method focuses on number of iteration and computational efficiency. The proposed model, is a variation of variational set level, peculiarly. We can extract the tumour itself without the background of the image. This can be done with inclusion of properties like solidity and eccentricity. It is suggested that along with energy other scale parameters should be considered which can help in increase the computational efficiency and improve the narrow band implementation.

As stated in the future work of the above algorithm the epsilon and sigma values are manipulated to find the tumour. This algorithm works with brain images which led to the decreasing of computational time and iterations. In the base paper the sigma value was taken as constant but the proposed method calculates the values of estimated sigma and simultaneously finds epsilon values which are based on density of the enclosing contour. Using this framework, the tumour is automatically segmented from the background and it decreases the iteration and CPU time.

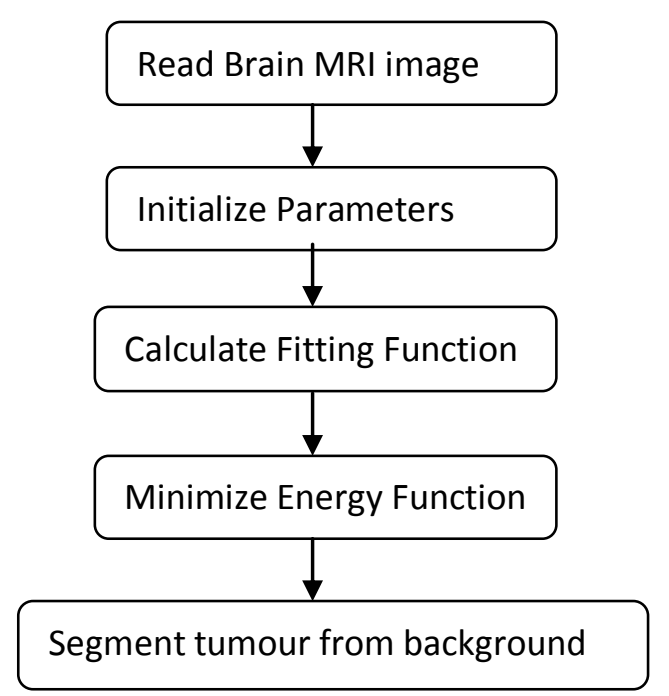

Figure 1: The Proposed Methodology

\section{EXPERIMENTAL RESULTS AND DISCUSSION}

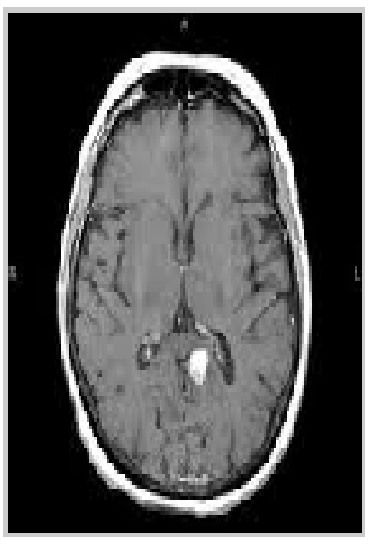

(a)

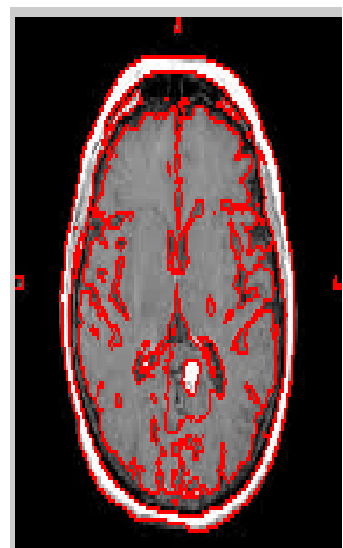

(b)
Figure 2: Results of the VLSF Method (a) Original Image (b) Final contour 150 iterations 


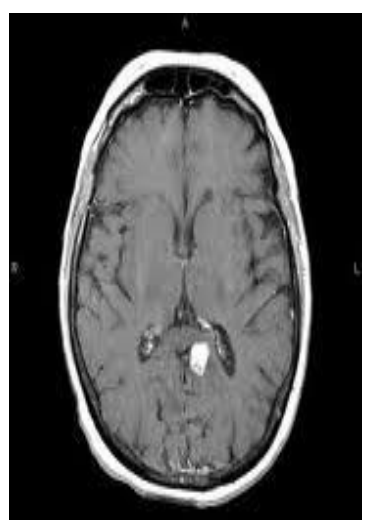

(a)

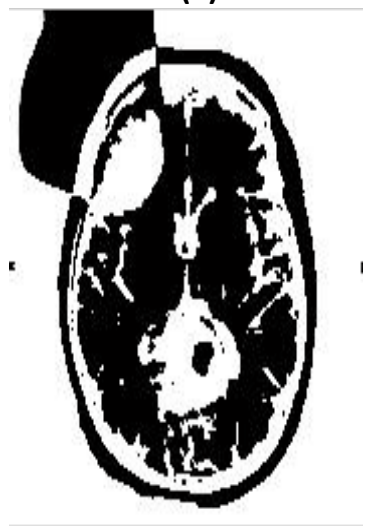

(c)

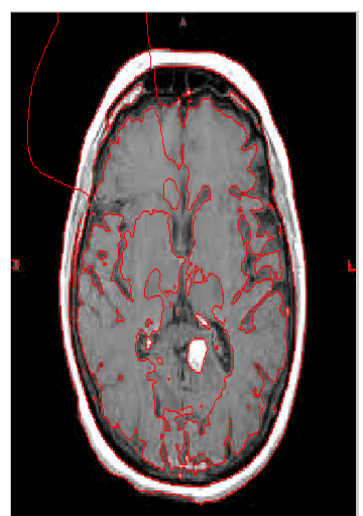

(b)

(d)
Figure 3: Results of the Proposed Method (a) original Image (b) and (c) Final contour 74 iterations (d) Tumor segmentation from background

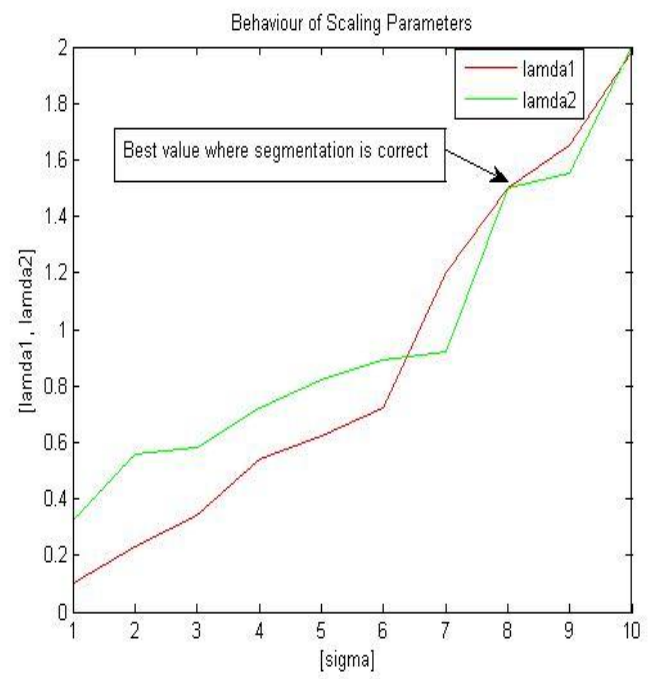

Figure 4: Graphical Results of the behavior of scaling Parameters.

The proposed model applied on both homogenous and nonhomogenous brain MRI images. The above figure 4 shows the behaviour of scaling parameters in segmentation of the brain MRI. In this paper, proposed method manipulates the various scaling parameters such as sigma, lamda1 and lamda2.
The value shown in the graph for sigma and lamdal and lamda2 are the best value where segmentation gives best results. The value obtained specifies the finer details of the object.

The result section gives information on the comparison done throughout the work. The VLSF segmentation method takes 150 iterations and the result is shown in fig 2(b). The proposed method takes 74 iterations and the result is shown in fig 3. The discussed algorithms were implemented in MATLAB. A Comparison of these algorithms is discussed in the below table 1 .

Table 1 Comparison of Segmentation algorithms.

\begin{tabular}{|l|l|l|}
\hline Parameters & $\begin{array}{l}\text { Variational Level } \\
\text { Set method[9] }\end{array}$ & $\begin{array}{l}\text { Proposed } \\
\text { Model }\end{array}$ \\
\hline Iterations & 150 & 74 \\
\hline $\begin{array}{l}\text { Noise } \\
\text { sensitivity }\end{array}$ & No & No \\
\hline $\begin{array}{l}\text { Time } \\
\text { Performance }\end{array}$ & It takes less time. & $\begin{array}{l}\text { It will takes } \\
\text { less time than } \\
\text { the other } \\
\text { model. }\end{array}$ \\
\hline $\begin{array}{l}\text { Intensity } \\
\text { Homogeneity }\end{array}$ & $\begin{array}{l}\text { Both } \\
\text { homogenous and } \\
\text { non homogenous }\end{array}$ & $\begin{array}{l}\text { Both } \\
\text { homogenous } \\
\text { and non } \\
\text { homogenous }\end{array}$ \\
\hline $\begin{array}{l}\text { Solidity \& } \\
\text { Eccentricity }\end{array}$ & $\begin{array}{l}\text { These properties } \\
\text { were not used in } \\
\text { this model }\end{array}$ & $\begin{array}{l}\text { These } \\
\text { properties are } \\
\text { used in this } \\
\text { model and } \\
\text { reduce the no } \\
\text { of iterations } \\
\text { and speed. }\end{array}$ \\
\hline
\end{tabular}

The tabulation given above shows the comparison between the Variational level Set method [9] and the proposed model. The comparison has been done in various paprameters such as number of iterations, time etc.

From the table 2 it is illustrated that the data for 5 patients has been collected from PGIMER, Chandigarh is used for segmentation. The tabulation given below shows the time of the segmentation of each image and the number of iterations taken by the segmentation algorithm. The table 2 gives the comparison of time and number of iterations between the Variational Level Set [9] and the proposed model. 
Table 2 Number of iterations and time taken by Segmentation Algorithms

\begin{tabular}{|c|c|c|c|c|}
\hline \multirow[b]{2}{*}{$\begin{array}{l}\text { No of } \\
\text { images }\end{array}$} & \multicolumn{2}{|l|}{ VLSF } & \multicolumn{2}{|c|}{$\begin{array}{l}\text { PROPOSED } \\
\text { METHOD }\end{array}$} \\
\hline & $\begin{array}{l}\text { Time } \\
(\mathrm{sec})\end{array}$ & $\begin{array}{l}\text { No of } \\
\text { iterations }\end{array}$ & $\begin{array}{l}\text { Time } \\
(\mathrm{sec})\end{array}$ & $\begin{array}{l}\text { No of } \\
\text { iterations }\end{array}$ \\
\hline 1 & $\begin{array}{r}13.2 \\
5\end{array}$ & 150 & 12.71 & 74 \\
\hline 2 & 7.8 & 120 & 6.92 & 60 \\
\hline 3 & 3.8 & 80 & 2.12 & 50 \\
\hline 4 & $\begin{array}{r}11.4 \\
7\end{array}$ & 100 & 9.68 & 74 \\
\hline 5 & 6.9 & 60 & 5.9 & 50 \\
\hline
\end{tabular}

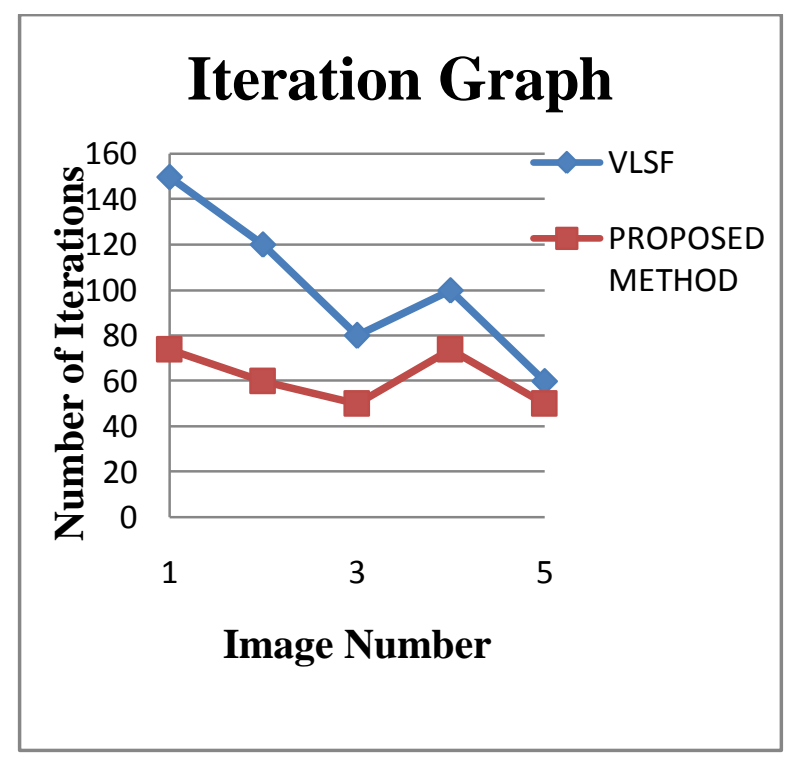

Figure 5: Graphical Result shows the iteration comparison between the VLSF and proposed.

The above figure 5 shows the iteration graph. As the iterative progress it passes through various stages of algorithm find the Neumann boundaries, inner and outer curvatures, and delta fitting function. Once the elimination of pixel occurs there is reduced number of sizes of the Neumann boundaries and the curvature leading to reduction in number of iteration. The proposed algorithm takes less number of iterations as compared to the original algorithm.

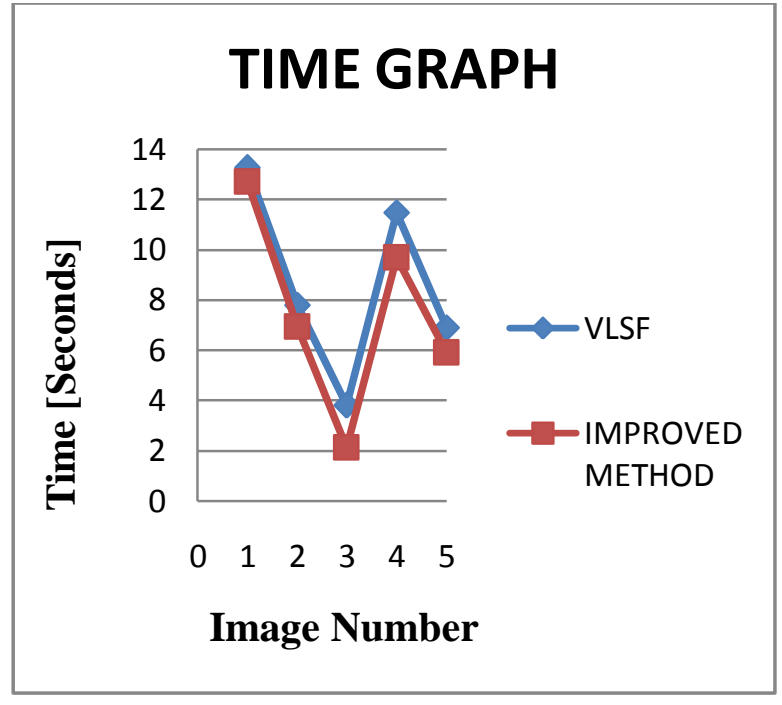

Figure 6: Graphical Result shows the time comparison between the VLSF and proposed.

The above figure 6 shows the time graph. The time consumed to process all the steps of algorithm have been analyzed and it has been found that as the proposed algorithm introduced the changed value of sigma, epsilon and lambda. The time consumption of proposed algorithm is less than the original algorithm.

\section{CONCLUSION}

In this paper, a method for segmenting the brain MRI has discussed. The original algorithm segments the whole brain MRI image but the proposed method segments the tumor from the background. Segmentation of the brain tumor for patients has shown in results and analysis by using the graph and tabulation method. This algorithm decreases the number of iteration and computation that are shown in the results and analysis. From the experimental results it has been concluded that this method segments the tumour from the background of the brain MRI. This work is really efficient platform for researchers and scientist. The proposed model is able to segment images with intensity inhomogeneity, and has desirable performance for images with weak object boundaries. Future Scope of the presented work is to segment the multiple junctions. This way the result may be more accurate.

\section{REFERENCES}

[1] Smitha Sunil Kumaran Nair and K. Revathy, "Quantitative analysis of brain tissues from Magnetic resonance images" IEEE International Conference on Digital Image Processing, 2009.

[2] V.Caselles, R. Kimmel, and G. Sapiro,"Geodesic active contour”,Int. J. Comput. Vis, vol. 22, pp.61-79, 1997.

[3] A.Vasilevskiy and K. Siddiqi, "Flux-maximizing geometric flows," IEEE Trans. Pattern Anal. Mach. Intell., vol. 24, no. 12, pp. 1565-1578, Dec.2002.

[4] T. Chan and L. Vese," Active contour without edges," IEEE trans Image Process., vol. 10, no. 2, pp 266-277, Feb. 2001.

[5] C. $\mathrm{Xu}$ and J. L. Prince, "Snakes, shapes, and gradient vector flow,"IEEE Trans. Image Process., vol.7, no. 3, pp.359-369,Mar.1998. 
[6] Jia Di, Yang Jin-Zhu, Zhang Yi-Fei. "An Efficient Modified Level Set Method For Brain Tissue Segmentation", IEEE International Conference on Information and Automation june 2010.

[7]. Shawn Lankton, Allen Tannenbaum, "Localizing RegionBased Active Contours," IEEE TRANSACTIONS ON IMAGE PROCESSING, VOL. 17, NO. 11, NOVEMBER 2008.

[8] Harman Kataria and Alka Jindal, " Brain MRI: Region based segmentation techniques" IJCST Vol. 3, Iss ue 1,
Jan. - March 2012 ISSN : 0976-8491 (Online) | ISSN : 2229-4333 (Print)

[9] Chunming Li ,Chiu-Yen Kao and John C.Gore,"Minimization of Region-Scalable Fitting Energy for Image Segmentation",IEEE Trans. On Image Processing, vol.17,n0. 10, October 2008.

[10] L. Vese and T. Chan, "A multiphase level set framework for image segmentation using the Mumford and Shah model, ’Int.J.Comput.Vis., vol. 50, pp. 271-293, 2002. 